\title{
Intermittent hypoxia mimicking obstructive sleep apnea aggravates early brain injury following ICH via neuroinflammation and apoptosis
}

\author{
WENJING FEI ${ }^{1}$, WEI JIAO ${ }^{2}$, XIAOYAN FENG $^{3}$, XUFENG CHEN $^{4}$ and YUHAI WANG ${ }^{3}$ \\ Departments of ${ }^{1}$ Neurology, ${ }^{2}$ Nursing, ${ }^{3}$ Neurosurgery and \\ ${ }^{4}$ Gastroenterology, 904th Hospital of Joint Logistic Support Force of PLA, Wuxi Clinical \\ College of Anhui Medical University, Wuxi, Jiangsu 214044, P.R. China
}

Received May 5, 2021; Accepted July 21, 2021

DOI: $10.3892 / \mathrm{mmr} .2021 .12464$

\begin{abstract}
Spontaneous intracerebral hemorrhage (ICH) is a subtype of stroke associated with high mortality and morbidity due to the lack of effective therapy. Obstructive sleep apnea (OSA) has been reported to aggravate early brain injury (EBI) and worsen the overall outcome of patients with $\mathrm{ICH}$. However, the precise role of OSA-mediated neuroinflammation and apoptosis following ICH has not been confirmed. The present study aimed to investigate the neuronal damage induced by OSA and the potential molecular mechanisms by which ICH-induced EBI regulates neural apoptosis in a C57BL/6 mouse ICH model. Mortality, neurological score, brain water content and neuronal death were evaluated by Evans blue extravasation, TUNEL staining, ELISA, analysis of reactive oxygen species/lipid peroxidation and western blotting. The results showed that OSA induction decreased survival rate, neurological score and neuron survival and upregulated the protein expression levels of Caspase-3, Bax, cytokines IL-1 $\beta$, IL- 6 and TNF- $\alpha$ and NF- $\kappa$ B, which indicated that OSA-mediated induction of apoptosis and neuroinflammation aggravated neuronal death following ICH. The molecular mechanism was partly dependent on the activating transcription factor/CHOP pathway. Taken together, the results demonstrated that OSA worsens neurological outcomes in mice and increases neuronal death by enhancing neural apoptosis and neuroinflammation.
\end{abstract}

Correspondence to: Professor Yuhai Wang, Department of Neurosurgery, 904th Hospital of Joint Logistic Support Force of PLA, Wuxi Clinical College of Anhui Medical University, 101 Xingyuan North Road, Wuxi, Jiangsu 214044, P.R. China E-mail: wangyuhai1516@163.com

Key words: obstructive sleep apnea, early brain injury, intracerebral hemorrhage, apoptosis, neuroinflammation

\section{Introduction}

Spontaneous intracerebral hemorrhage (ICH) has the highest mortality rate of $\sim 40 \%$ worldwide among stroke subtypes (1), accounts for $15-20 \%$ of all stroke cases, and is more common in elderly patients (2-4). Acute ICH due to large intracranial hematoma is associated with high morbidity and mortality as it can lead to primary brain injury via destruction of brain tissue and high intracranial pressure (ICP) (5-7). Previous studies revealed that craniotomy for hematoma evacuation is an effective therapy for limiting primary brain damage and decreasing ICP following ICH $(5,8,9)$. However, long-term outcomes were found to not be altered, and it rarely affects neurological recovery (10). Increasing numbers of studies have found that red blood cell debris (hemoglobin and its degradation products) and other blood components trigger secondary brain injury following $\mathrm{ICH}$ and contribute to a series of damaging events, including neuroinflammation, brain edema, oxidative stress, blood-brain barrier (BBB) damage and neuronal death (11-16). An increasing number of studies has been investigated the mechanisms underlying ICH-induced secondary injury to identify improved therapeutic targets for $\mathrm{ICH}$; the potential mechanisms underlying EBI include autophagy, apoptosis, direct neuronal death and necroptosis $(13,17,18)$.

Numerous factors, including hypertension, obstructive sleep apnea (OSA), smoking, obesity and hyperlipidemia, can induce intracranial aneurysms, promote aneurysm rupture, aggravate early brain injury (EBI) and worsen the overall outcome of patients with vascular aneurysm following hemorrhagic stroke (19-21). Geer et al (22) also reported that the incidence rate of hypertension, heart disease and hyperlipidemia, as well as body mass index, were higher in patients with OSA than in those without. A multivariable logistic regression model showed that OSA is associated with an increased risk of ICH in a recent study (23). Pontes-Neto et al (24) also found that the incidence of OSA is higher in patients with $\mathrm{ICH}$ and may aggravate perihematomal edema.

The mechanism by which OSA aggravates brain injury is unclear. Orrù et al (25) reported that OSA induces oxidative stress and inflammation and disrupts vascular function by releasing excessive levels of NO and its derivatives. Apoptosis 
and neuroinflammation are involved in hypoxia-induced cell death and tissue injury, especially in OSA and hypoxia-associated disease, such as intermittent hypoxia (26) and cerebral ischemia (27). Previous studies have reported that activating transcription factor (ATF)4 is a transcriptional regulation factor that serves an important role in $\mathrm{ICH}$ and that the ATF4/CHOP signaling pathway regulates cell death via aggravated neuroinflammation and apoptosis $(28,29)$. To the best of our knowledge, however, the effect of OSA in ICH has not been investigated and the specific mechanism is unclear. The present study aimed to investigate the neuronal damage induced by OSA and the potential molecular mechanisms by which ICH-induced EBI regulates neural apoptosis.

\section{Materials and methods}

Animals. All animal experiments complied with the National Institutes of Health guidelines (30) for the handling of laboratory animals and were approved by the Ethics Committee of the Wuxi Clinical College of Anhui Medical University (approval no. YXLL-2020-112; Wuxi, China). All experiments were performed on 159 healthy adult male C57BL/6J mice (age, 6-8 weeks; weight, 22-25 g; Anhui Medical University). Mice were divided into the following groups ( $n=15 /$ group): Sham, OSA, ICH, ICH + OSA, ICH + small interfering (si)-control (Con), ICH + si-ATF4, ICH + OSA + si-Con and ICH + OSA + si-ATF4. Overall, there were 16 mice in the Sham group (one dead), 16 mice in the OSA group (one dead), 19 mice in the $\mathrm{ICH}$ group (four dead), 23 mice in the $\mathrm{ICH}+\mathrm{OSA}$ group (eight dead), 20 mice in the $\mathrm{ICH}+$ si-Con (five dead), 18 mice in the ICH + si-ATF4 (three dead), 25 mice in the ICH + OSA + si-Con (10 dead), 22 mice in the ICH + OSA + si-ATF4 (seven dead). The mice were housed in a climate-controlled environment at $25 \pm 2^{\circ} \mathrm{C}$ and $55 \pm 5 \%$ humidity with 12 -h light/dark cycles, and had free access to food and water.

ICH animal model. The ICH mouse model was constructed via autologous blood injection, as previously described (31). Briefly, male C57BL6/J mice were anesthetized by intraperitoneal (i.p.) injection of $50 \mathrm{mg} / \mathrm{kg}$ pentobarbital sodium and placed in a prone position with a stereotactic head frame. The rectal temperature was maintained at $37.0 \pm 0.5^{\circ} \mathrm{C}$ during the operation with a heating pad. An artificial tear ointment was used to protect the eye from injury during surgery. A midline scalp incision was made and a cranial burr hole $(1 \mathrm{~mm}$ diameter) was made at the following coordinates relative to bregma: 0.2 posterior, 2.2 lateral and $3.5 \mathrm{~mm}$ below the dura. A total of $30 \mu \mathrm{l}$ autologous blood without anticoagulation was collected from the caudal artery and rapidly injected into the basal ganglia via the burr hole using the 26 gauge needle of a 10- $\mu$ l Hamilton syringe. First, $5 \mu \mathrm{l}$ arterial blood was injected at a depth of $2.8 \mathrm{~mm}$ from the dura (injection speed, $3 \mu \mathrm{l} / \mathrm{min}$ ). After $5 \mathrm{~min}$, the remaining $25 \mu \mathrm{l}$ blood was injected at a depth of $3.5 \mathrm{~mm}$ (injection speed, $3 \mu \mathrm{l} / \mathrm{min}$ ). Following the injection of autologous blood, the needle was left in the brain for $10 \mathrm{~min}$ to prevent blood backflow along the needle tract. Finally, the hole was covered with medical bone wax. The animals in the Sham group underwent the same surgical procedures but were injected at the same sites with an equal volume of $0.9 \%$ sterile saline instead of blood.
Intermittent hypoxia (IH) model. An IH model was used to construct the OSA model as previously described (32). At 30 min after recovery from anesthesia, mice in the OSA group were exposed to air for $90 \mathrm{sec}$, followed by $90 \mathrm{sec}$ of progressive hypoxia to a nadir of $5 \%$ inhaled $\mathrm{O}_{2}$ to model moderate SA. The mice were exposed to IH for $7 \mathrm{~h}$ /day during the light period (10 a.m. to 5 p.m.) for 3 consecutive days. During the hypoxia phase, the $\mathrm{O}_{2}$ concentration in the chamber was decreased to $5 \%$ within $20 \mathrm{sec}$ by infusion of $\mathrm{N}_{2}$ and remained at that concentration for $90 \mathrm{sec}$. Then, the $\mathrm{O}_{2}$ concentration was rapidly increased to $21 \%$ within $10 \mathrm{sec}$ by flushing the chamber with compressed clean air, which was sustained for $90 \mathrm{sec}$. Following IH induction, the mice were transferred to the normal housing environment with room air, neurological score was measured and mice were euthanized for brain tissue collection.

siRNA treatment and transfection. The mice were anesthetized with pentobarbital sodium $(50 \mathrm{mg} / \mathrm{kg})$ and placed on stereotaxic apparatus (Narishige International Ltd.). Then, a burr hole was made in the left hemisphere at the following coordinates: 0.2 posterior, 1.0 lateral and $2.2 \mathrm{~mm}$ below the horizontal plane of the bregma. A total of $5 \mu 1$ siRNA was injected into the left lateral ventricle at a rate of $0.5 \mu 1 / \mathrm{min}$. Lipofectamine $^{\mathrm{TM}}$ RNAiMax reagent (Invitrogen; Thermo Fisher Scientific, Inc.) was used in Opti-MEM medium, according to the manufacturer's instructions. To enhance the silencing effect, the injection was performed $48 \mathrm{~h}$ before ICH. Targeted and control siRNAs were synthesized by Shanghai GeneChem Co., Ltd., as follows: si-ATF4 forward, 5'-GUG AGAAACUGGAUAAGAATT-3' and reverse, 5'-UUCUUA UCCAGUUUCUCACTT-3' and negative control siRNA forward, 5'-UUCUCCGAACGUGUCACGUTT-3' and reverse, 5'-ACGUGACACGUUCGGAGAATT-3'.

Neurobehavioral and mortality assessment. The severity of brain injury was evaluated by determining the neurological function $72 \mathrm{~h}$ after ICH as previously described (17). The scoring system consisted of six tests, and specific standards are shown in Table SI. The final neurological score ranged from 3 to 18 and included spontaneous activity (0-3), movement symmetry of all limbs (0-3), forelimb outstretching (0-3), body proprioception (1-3), response to vibrissae touch (1-3) and climbing (1-3). A total of 10 mice in all groups underwent neurobehavioral assessment, and a higher score represented improved neurological function. Following the establishment of the ICH/OSA model and the neurobehavioral assessment, mortality assessment was performed and death was confirmed. Dead animals were replaced to ensure 15 mice in each group. The mortality was defined as the ratio of dead mice to the total number in each group.

Brain water content measurement. At $72 \mathrm{~h}$ post-ICH, the mice were sacrificed with $100 \mathrm{mg} / \mathrm{kg}$ sodium pentobarbital via i.p. injection. Death was confirmed by cessation of breathing and corneal reflex, then brain tissue samples were collected. The severity of brain edema was evaluated by measuring the brain water content using the standard wet-dry method, as previously reported $(17,27,33)$. The entire brain was harvested and separated into the ipsilateral and contralateral cortex and 
basal ganglia and cerebellum (wet weight). Then, brain specimens from each group were dehydrated at $105^{\circ} \mathrm{C}$ for $24 \mathrm{~h}$ to acquire the dry weight. The percentage of brain water content was calculated as follows: (Wet weight-dry weight)/wet weight $\mathrm{x} 100 \%$.

Evans blue extravasation. Evans blue extravasation was performed as previously described (34). Briefly, all mice were anesthetized with intraperitoneal (i.p.) pentobarbital sodium $(50 \mathrm{mg} / \mathrm{kg})$ injection. Evans blue dye $(2 \% ; 5 \mathrm{ml} / \mathrm{kg}$; Sigma-Aldrich; Merck KGaA) was injected into the left femoral vein for $>2 \mathrm{~min}$ and allowed to circulate for $60 \mathrm{~min}$. Then, mice were sacrificed with $100 \mathrm{mg} / \mathrm{kg}$ sodium pentobarbital via i.p. injection followed by phosphate-buffered saline (PBS) intracardial perfusion. Death was confirmed by cessation of breathing and corneal reflex. The brains were removed and divided into the left and right cerebral hemispheres, weighed, homogenized in saline and centrifuged at 15,000 x g for $30 \mathrm{~min}$ at room temperature. The supernatant was added to an equal volume of trichloroacetic acid, incubated overnight at $4^{\circ} \mathrm{C}$ and centrifuged at $15,000 \mathrm{x} \mathrm{g}$ for $30 \mathrm{~min}$ at room temperature. The supernatant was collected and spectrophotometrically quantified at $610 \mathrm{~nm}$ to measure the amount of Evans blue dye.

TUNEL staining. TUNEL and neuronal nuclei (NeuN) co-staining were performed to assess neuronal death in the brain cortex and were fixed with $4 \%$ paraformaldehyde for $1 \mathrm{~h}$ at $25^{\circ} \mathrm{C}$. Paraffin-embedded sections $(10 \mu \mathrm{m})$ were cut from formalin-fixed tissue and stained with TUNEL and NeuN stain. TUNEL reaction mixture $(50 \mu \mathrm{l})$ was added to each sample and slides were incubated in a humidified dark chamber for $60 \mathrm{~min}$ at $37^{\circ} \mathrm{C}$. Then, a primary antibody against NeuN (1:200; rabbit polyclonal; cat. no. ab128886; Abcam) diluted in PBS was added, followed by incubation overnight at $4^{\circ} \mathrm{C}$. The slides were then incubated with DAPI $(0.1 \mathrm{mg} / \mathrm{ml})$ for $5 \mathrm{~min}$ at room temperature in the dark to stain the nuclei, followed by imaging with a fluorescence microscope (magnification, $\mathrm{x} 400$ ). The procedure was performed according to the manufacturer's instructions with a TUNEL staining kit (cat. no. 1684817; Roche Diagnostics GmbH). A negative control (without the TUNEL reaction mixture) was used. The apoptotic index (\%) was calculated as follows: Number of TUNEL-positive cells/total number of cells $x 400$. The cell count was confirmed in four randomly selected high-power fields and the data were averaged.

Cytokine measurement. Hippocampal levels of cytokines were detected by ELISA. Briefly, hippocampal samples were collected and dissolved using RIPA buffer (CoWin Biosciences), then cell lysate was centrifuged for 3-5 min at $12,500 \mathrm{x} \mathrm{g}$ at room temperature and the supernatant was collected. IL-1 $\beta$ (cat. no. ab197742), IL-6 (cat. no. ab222503), TNF- $\alpha$ (cat. no. ab208348) and NF- $\kappa$ B (cat. no. ab176663) were measured by ELISA according to the manufacturer's instructions (all Abcam).

Reverse transcription-quantitative $(R T-q) P C R$. RT-qPCR analysis was performed as described previously (35). Total RNA was extracted from hippocampal brain samples using TRIzol (Invitrogen; Thermo Fisher Scientific, Inc.) according to the manufacturer's instructions. Then, the RNA was reverse-transcribed into complementary DNA using the RevertAid First Strand cDNA Synthesis kit (cat. no. K1622; Thermo Fisher Scientific Inc.). The ATF4 and CHOP mRNA levels in each sample were measured by qPCR using SYBR-Green Master Mix (Toyobo Life Science). GAPDH was used as an internal control. The qPCR thermocycling conditions were as follows: Initial denaturation at $45^{\circ} \mathrm{C}(2 \mathrm{~min})$ and $95^{\circ} \mathrm{C}(10 \mathrm{~min})$, followed by 40 cycles of denaturation at $95^{\circ} \mathrm{C}(15 \mathrm{sec})$, annealing at $60^{\circ} \mathrm{C}(1 \mathrm{~min})$ and extension at $72^{\circ} \mathrm{C}(1 \mathrm{~min})$. The $2^{-\Delta \Delta \mathrm{Cq}}$ method was used to assess the relative mRNA expression levels (36). All samples were analyzed in triplicate. The primers used are listed as follows: ATF4 forward, 5'-ATGACCGAAATGAGC TTCCTG-3' and reverse, 5'-GCTGGAGAACCCATGAGGT-3'; CHOP forward, 5'-GGAAACAGAGTGGTCATTCCC-3' and reverse, 5'-CTGCTTGAGCCGTTCATTCTC-3'; and GAPDH forward, 5'-ATGGGTGTGAACCACGAGA-3' and reverse, 5'-CAGGGATGATGTTCTGGGCA-3'.

Western blot analysis. Western blot analysis was performed as described previously (33). Briefly, cerebral cortex samples were collected, homogenized and total protein was extracted using RIPA buffer (CoWin Biosciences). A BCA Protein Assay kit (Beyotime Institute of Biotechnology) was used to measure the protein concentration. Total protein $(30 \mu \mathrm{g})$ was separated via $12 \%$ SDS-PAGE and transferred onto PVDF membranes. The membranes were blocked at room temperature for $1 \mathrm{~h}$ with $5 \%$ non-fat milk and incubated with primary antibodies (all Abcam) overnight at $4^{\circ} \mathrm{C}$ as follows: Rabbit anti- $\beta$-actin $(1: 1,000$; cat. no. ab8227), anti-Caspase-3 (1:2,000; cat. no. ab184787), anti-Bax (1:2,000; cat. no. ab182733), anti-Bcl2 (1:2,000; cat. no. ab182858), anti-ATF4 (1:1,000; cat. no. ab216839) and mouse anti-CHOP (5 $\mu \mathrm{g} / \mathrm{ml}$; cat. no. ab11419). After washing the membranes with TBS with $0.5 \%$ Tween-20 three times, horseradish peroxidase-conjugated anti-rabbit (1:2,000; cat. no. 7074S; Cell Signaling Technology, Inc.) or anti-mouse IgG (1:2,000; cat. no. 7076S; Cell Signaling Technology, Inc.) secondary antibodies were applied and incubated at room temperature for $1.5 \mathrm{~h}$. The signals were developed using an enhanced chemiluminescence reagent (MilliporeSigma) according to the manufacturer's instructions. The protein bands were detected using a Bio-Rad imaging system (Bio-Rad Laboratories, Inc.) and quantified with ImageJ (version 1.52; National Institutes of Health).

Statistical analysis. All experiments were repeated $>3$ times and data are presented as the mean \pm SEM. SPSS 14.0 (SPSS, Inc.) and GraphPad Prism 6 (GraphPad Software, Inc.) were used for statistical analysis. Student's t test (unpaired, two-tailed) was used to analyze the statistical differences between two groups. Differences between multiple groups were analyzed using one-way ANOVA followed by post hoc Tukey's test. $\mathrm{P}<0.05$ was considered to indicate a statistically significant difference.

\section{Results}

Mortality and neurological function in ICH/OSA model mice. Previous clinical studies reported that OSA aggravates EBI, increases mortality rate and worsens overall outcomes 

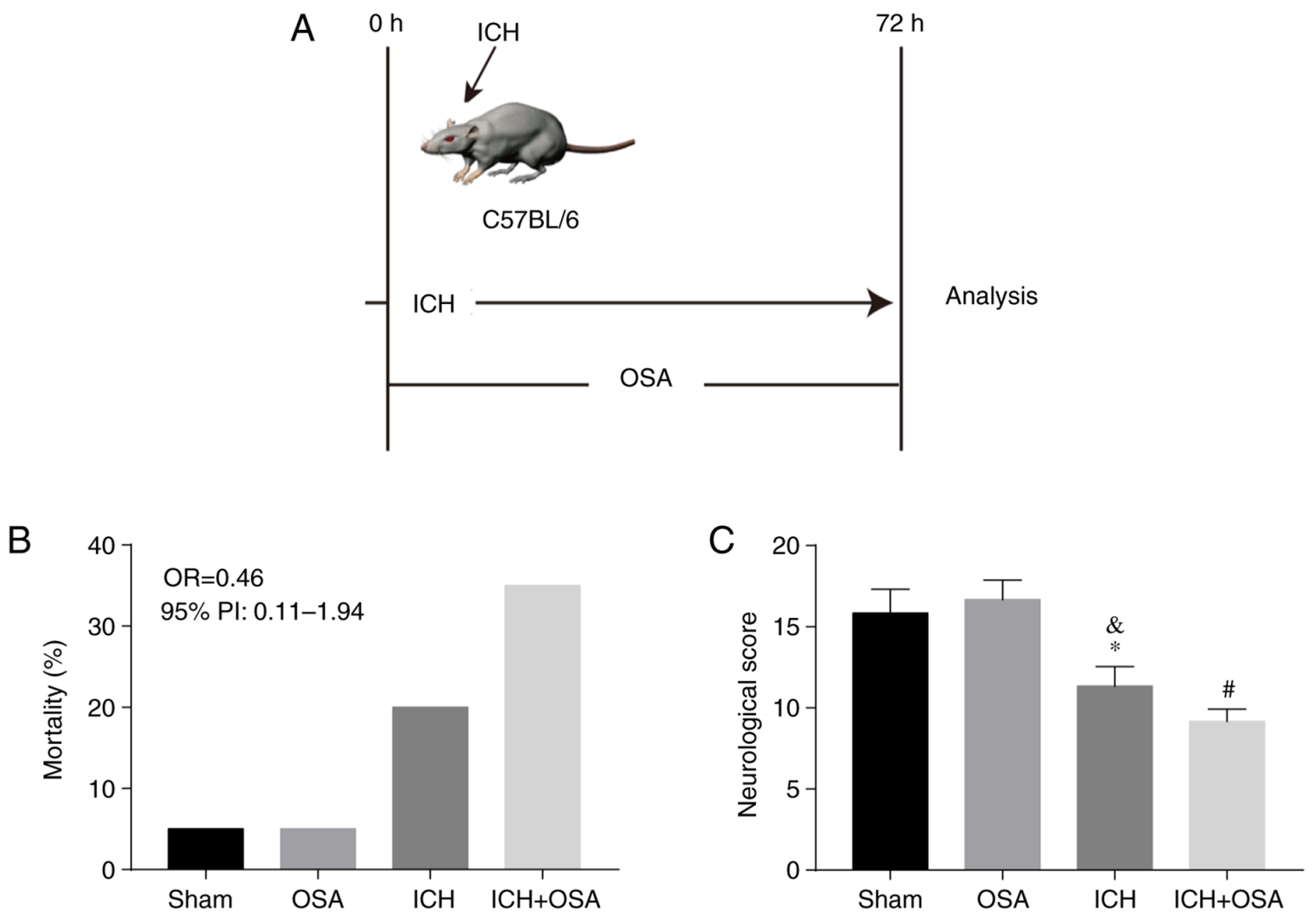

Figure 1. Mortality and neurological function in ICH/OSA mouse models. (A) Schematic of the experimental paradigm for modeling ICH/OSA in mice. (B) Mortality rate increased in the ICH + OSA group but there was no significant difference compared with the ICH group. (C) Neurological score of mice after $72 \mathrm{~h}$ (n=10). Data are presented as the mean \pm SEM. ${ }^{*} \mathrm{P}<0.05$ vs. Sham; ${ }^{\circledR} \mathrm{P}<0.05$ vs. OSA; $\mathrm{P}<0.05$ vs. ICH. ICH, intracerebral hemorrhage; OSA, obstructive sleep apnea.

of patients with $\mathrm{ICH}(22,37)$. Thus, an ICH model was constructed and chronic (C)IH was used to construct the OSA model (Fig. 1A). The effect of OSA on mortality rate and neurological score was assessed. There were 16 mice in the Sham group (one dead), 16 mice in the OSA group (one dead), 19 mice in the ICH group (4 dead) and 23 mice in the $\mathrm{ICH}+$ OSA group (8 dead). Mortality rate (Fig. 1B) increased in the subarachnoid hemorrhage $(\mathrm{ICH})+$ OSA group but there was no significant difference compared with the ICH group (OR=0.46; 95\% PI, 0.11-1.94). Neurological score decreased significantly following $\mathrm{ICH}$ and was further aggravated by OSA (Fig. 1C).

OSA aggravates EBI and BBB permeability following $I C H$. To assess EBI following ICH and OSA, brain water content was measured by the wet-dry method at $72 \mathrm{~h}$ post-ICH to evaluate the degree of brain damage. The results showed that ICH significantly increased the brain water content in the ipsilateral cortex and both ipsilateral and contralateral basal ganglia; OSA significantly aggravated this in the ipsilateral cortex and basal ganglia (Fig. 2A). Similar results were observed for BBB permeability, which increased significantly following ICH and was significantly aggravated by OSA (Fig. 2B). To assess hippocampal neuronal death following ICH and OSA, TUNEL assay was performed. As expected, more hippocampal neuron death was observed in the ICH + OSA group compared with in the ICH group (Fig. 2C). Hence, these data showed that OSA aggravates EBI following $\mathrm{ICH}$.
OSA aggravates neuroinflammation following ICH. Neuroinflammation serves a key role in EBI following ICH; increased neuroinflammation aggravates EBI $(27,38)$. The inflammatory complex induces secretion of pro-inflammatory cytokines, including IL-1 $\beta$, IL-6, TNF- $\alpha$ and NF- $\kappa \mathrm{B}(27)$. Therefore, hippocampal levels of IL-1 $\beta$, IL-6, TNF- $\alpha$ and $\mathrm{NF}-\kappa \mathrm{B}$ were assessed by ELISA. The results showed that expression levels of pro-inflammatory cytokines increased significantly following ICH and were further increased in the OSA + ICH group (Fig. 3A-D). The results showed that OSA aggravated EBI in a mouse model of ICH and that the mechanism may be partly associated with the activation of neuroinflammation.

OSA further increases expression levels of apoptosisassociated proteins following ICH. Previous studies have indicated that apoptosis is an important form of cell death in central nervous system (CNS) disease and serves a key role in SAH and OSA $(26,27)$. Expression levels of apoptosis-associated proteins Caspase-3, Bax, and Bcl-2 following ICH and OSA were evaluated by western blotting (Fig. 4A). The results showed that expression levels of Caspase-3 and Bax increased significantly following ICH and were further increased by subsequent OSA. Protein expression levels of anti-apoptotic Bcl-2 were significantly decreased by $\mathrm{ICH}$ and further decreased in the ICH + OSA group (Fig. 4B-D). The results showed that OSA aggravated EBI in a mouse model of ICH and that the mechanism may be partly associated with the activation of apoptosis. 
A

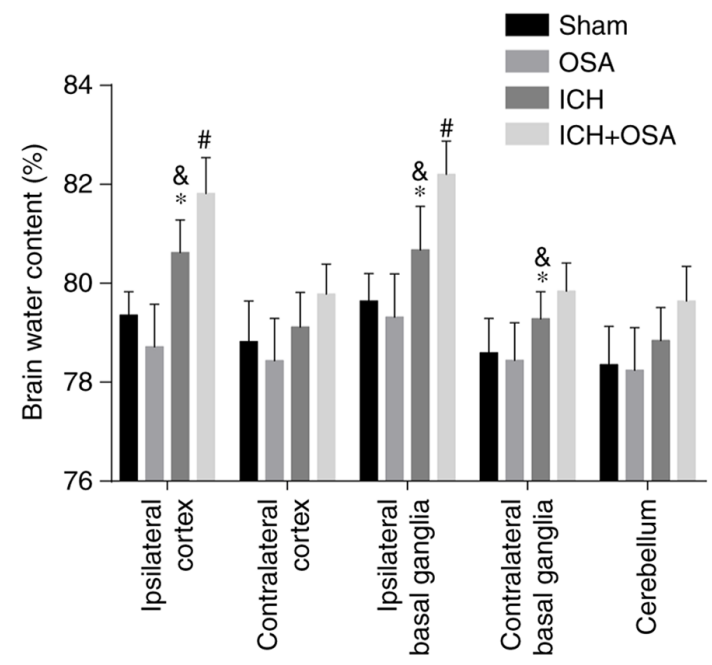

C

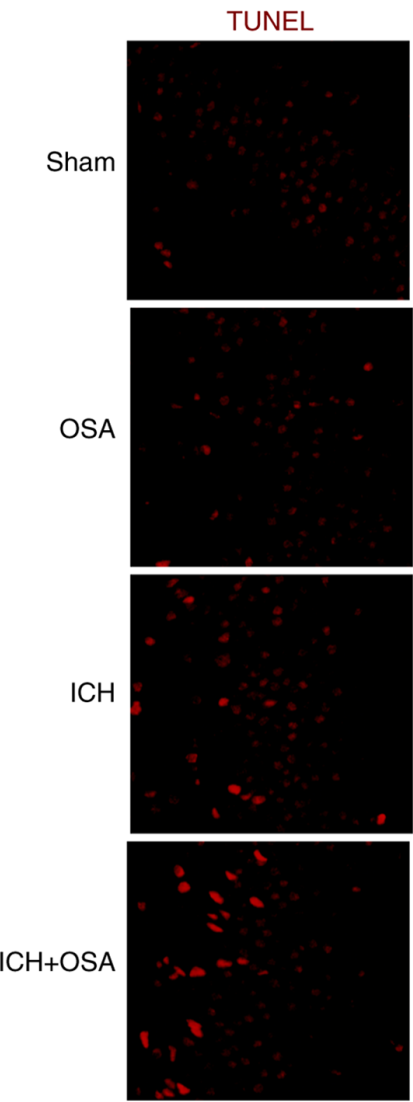

DAPI
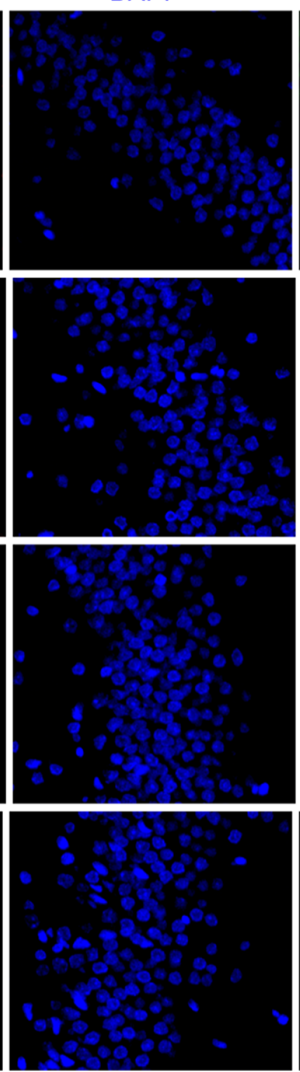

B

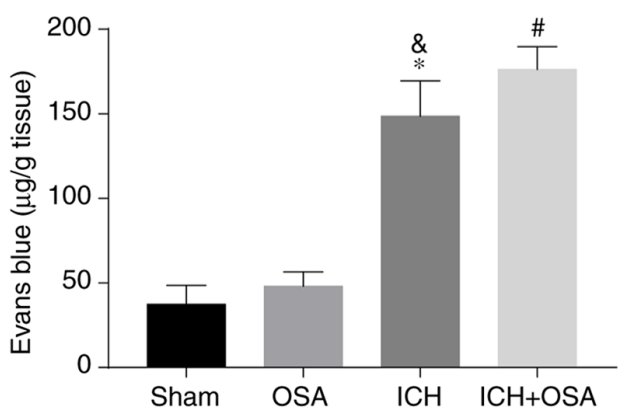

Figure 2. OSA aggravates early brain injury and BBB permeability following ICH. (A) ICH and subsequent OSA increased brain water content in the ipsilateral cortex and ipsilateral and contralateral basal ganglia. (B) OSA increased BBB permeability following ICH. (C) TUNEL staining of apoptotic neurons. OSA aggravated neuronal apoptosis in the hippocampus post-ICH. Scale bar, $50 \mu \mathrm{m}$. Data are presented as the mean $\pm \mathrm{SEM}$. ${ }^{*} \mathrm{P}<0.05 \mathrm{vs}$. Sham; ${ }^{\circledR} \mathrm{P}<0.05 \mathrm{vs}$. OSA; ${ }^{\text {"P }}<0.05$ vs. ICH. OSA, obstructive sleep apnea; BBB, blood-brain barrier; ICH, intracerebral hemorrhage; NeuN, neuronal nuclei.

OSA aggravates EBI via the ATF4/CHOP signaling pathway following ICH. Deng et al (39) reported that the ATF4/CHOP signaling pathway is a key mechanism by which brain injury is ameliorated in ICH rats. Therefore, the present study investigated whether OSA aggravated apoptosis and neuroinflammation by regulating the ATF4/CHOP signaling pathway. First, protein and gene expression levels of ATF4 and CHOP were assessed following ICH and OSA by western blotting and RT-qPCR. The results showed that mRNA expression levels of ATF4 and CHOP increased following $\mathrm{ICH}$ and further increased following OSA
(Fig. 5A and B). Similar results were demonstrated by western blot analysis of protein expression levels (Fig. 5C-E). Hence, it was hypothesized that OSA aggravated EBI by inhibiting the ATF4/CHOP signaling pathway following ICH.

Knockdown of ATF4 reverses OSA-aggravated EBI following $I C H$. To investigate the role of ATF4 in EBI, transfection with targeted siRNA (si-ATF4) was performed to downregulate expression of ATF4. There was no significant difference in mortality rate following ATF4 knockdown(Fig. 6A).RT-qPCR 
A

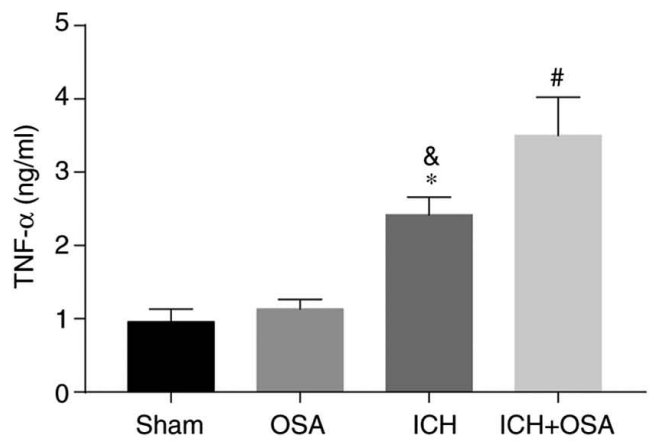

C

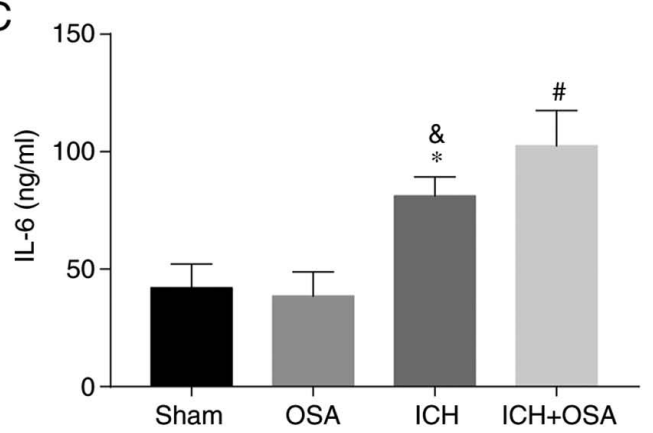

$\mathrm{B}$
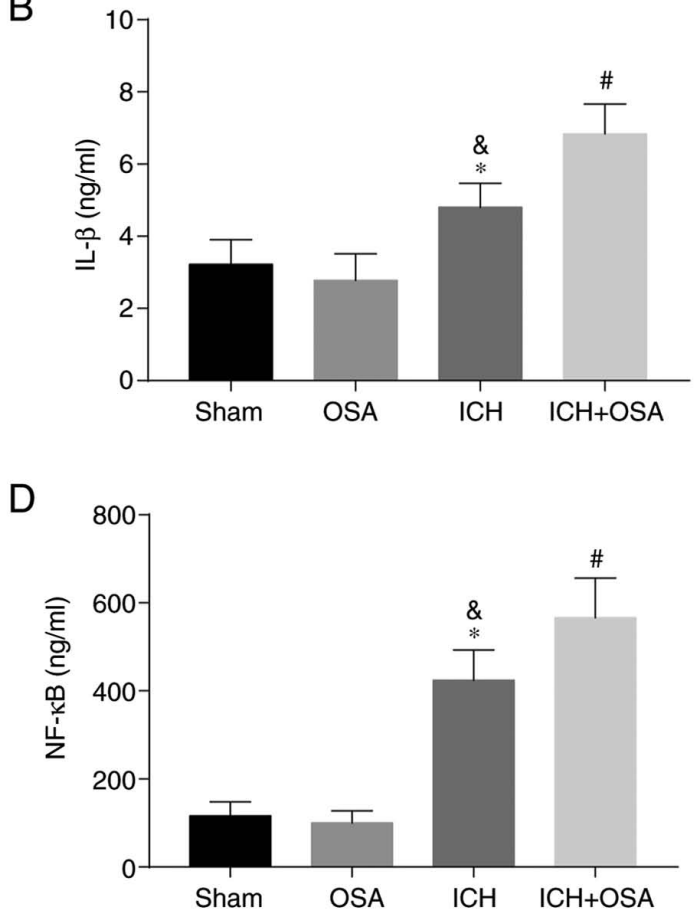

Figure 3. OSA aggravates neuroinflammation following ICH. Following ICH, OSA significantly aggravated hippocampal expression levels of (A) TNF- $\alpha$, (B) IL-1 $\beta$, (C) IL-6 and (D) NF-kB. Data are presented as the mean \pm SEM $(n=5)$. "P<0.05 vs. Sham; ${ }^{\circ} \mathrm{P}<0.05$ vs. OSA; ${ }^{*} \mathrm{P}<0.05$ vs. ICH. OSA, obstructive sleep apnea; $\mathrm{ICH}$, intracerebral hemorrhage.

A

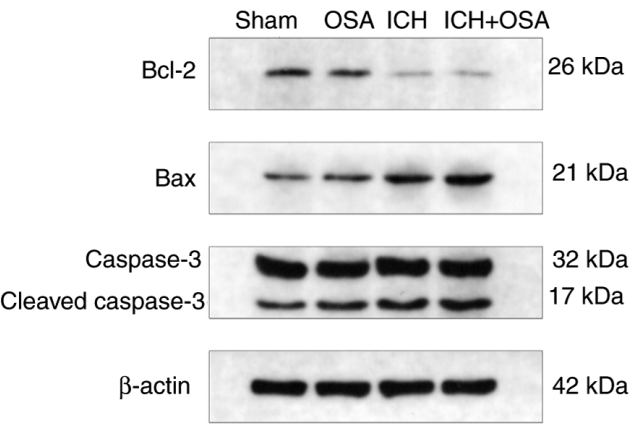

C

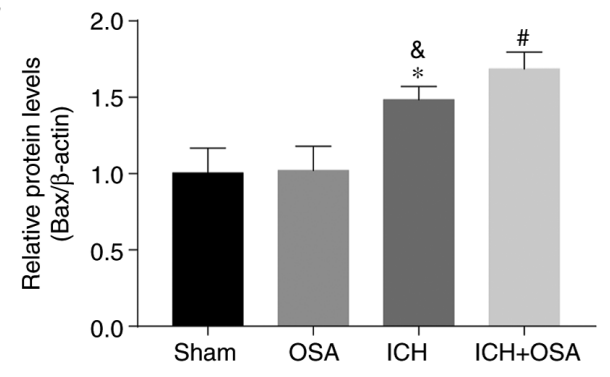

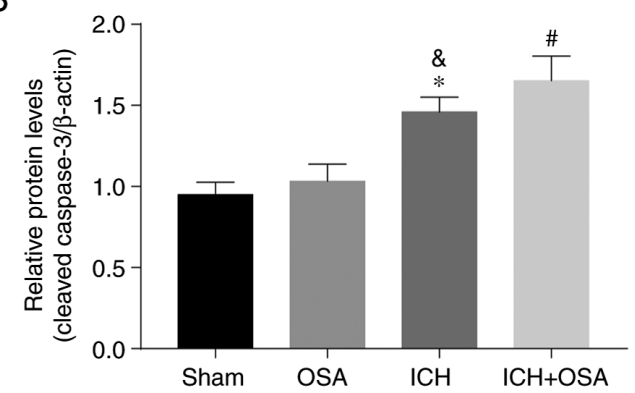

D

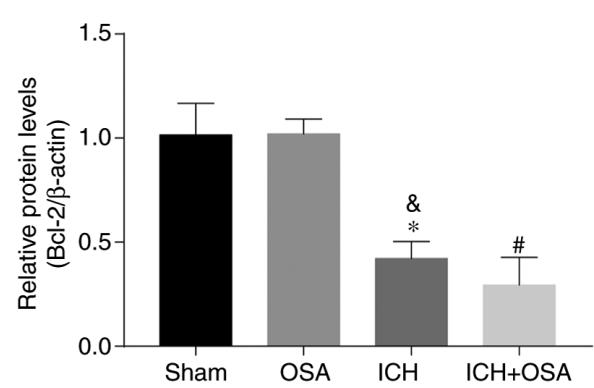

Figure 4. OSA increases expression levels of apoptosis-associated proteins following ICH. (A) Expression levels of Caspase-3, Bax and Bcl-2 in the brain cortex of mice were assessed following ICH/OSA by western blotting. Expression levels of (B) Cleaved-Caspase-3, (C) Bax and (D) Bcl-2 in the brain cortex relative to $\beta$-actin loading control. OSA significantly increased Cleaved-Caspase-3 and Bax expression levels and decreased those of anti-apoptosis protein Bcl-2 following ICH. Data are presented as the mean \pm SEM $(n=5)$. ${ }^{*} \mathrm{P}<0.05$ vs. Sham; ${ }^{\circledR} \mathrm{P}<0.05$ vs. OSA; ${ }^{\prime \prime} \mathrm{P}<0.05$ vs. ICH. OSA, obstructive sleep apnea; $\mathrm{ICH}$, intracerebral hemorrhage.

was used to confirm that transfection was successful and ATF4 expression was efficiently silenced (Fig. S1). Following ATF4 knockdown, neurological score, ipsilateral basal ganglia brain edema and BBB permeability were improved (Fig. 6B-D), indicating a reversal of OSA-induced EBI. The levels of apoptosis-associated proteins (Cleaved-Caspase-3, Bcl-2 and Bax) were assessed by western blotting (Fig. 6E). The expression levels of Cleaved-Caspase- 3 and Bax were decreased and those of $\mathrm{Bcl}-2$ increased significantly following ATF4 knockdown (Fig. 6F-H). 

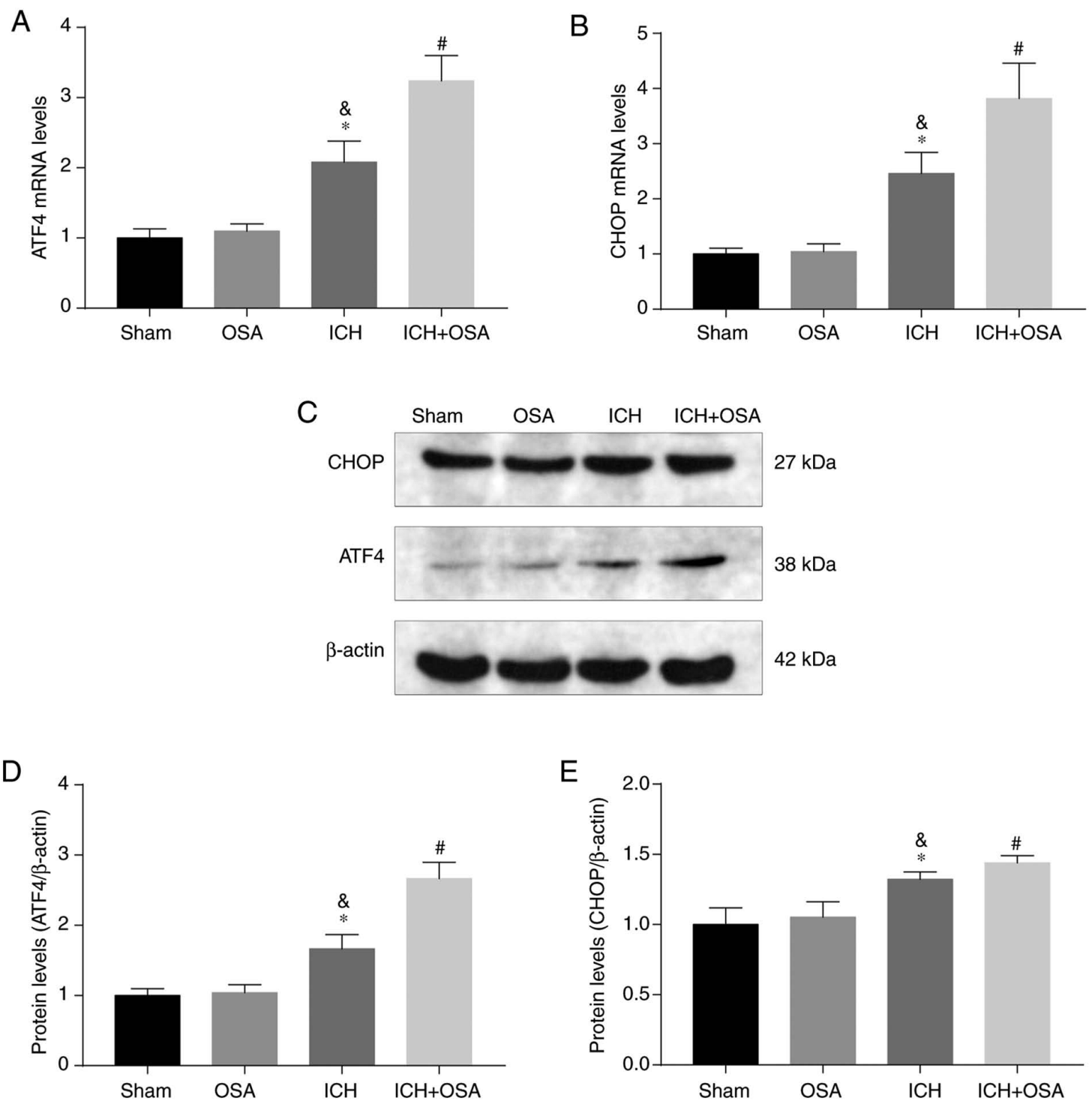

Figure 5. OSA aggravates early brain injury via the ATF4/CHOP signaling pathway following ICH. mRNA expression of (A) ATF4 and (B) CHOP in the brain cortex of mice following ICH/OSA was assessed by reverse transcription-quantitative PCR. OSA significantly increased mRNA expression of ATF4 and CHOP following ICH. (C) Protein expression levels of ATF4 and CHOP in the brain cortex of mice following ICH/OSA were assessed by western blotting. OSA significantly increased the protein expression levels of (D) ATF4 and (E) CHOP following ICH. Data are presented as the mean \pm SEM ( $=5$ ). ${ }^{*} \mathrm{P}<0.05$ vs. Sham; ${ }^{\&} \mathrm{P}<0.05$ vs. OSA; ${ }^{\text {P }}<0.05$ vs. ICH. ATF4, activating transcription factor 4; OSA, obstructive sleep apnea; ICH, intracerebral hemorrhage.

\section{Discussion}

The present study evaluated the mechanisms by which OSA aggravates EBI in a mouse ICH model. The present study demonstrated that OSA aggravates EBI, neurological dysfunction, brain damage, neuroinflammation, apoptosis and neuronal death following ICH. The potential mechanism may be associated with the ATF4/CHOP signaling pathway.

OSA is characterized by repetitive narrowing or collapsing of the upper airways, resulting in recurrent hypoxia during sleep (40). Interruption of breathing by OSA results in $\mathrm{IH}$, leading to decreased blood oxygen saturation and impaired sleep quality; prolonged hypoxia induces inflammatory responses, which affect the function of the vascular endothelium (41). OSA not only seriously affects the quality of life but also induces or aggravates various comorbidities, including hypertension (40), coronary heart disease (42), ICH (25) and stroke (43). The potential molecular mechanisms of OSA-induced disease are complicated. OSA induces oxidative stress and activation of the inflammatory system, resulting in the production of reactive oxygen species (ROS); ROS may be a potential therapeutic target for ameliorating hypoxia-induced cell death and tissue injury (44). Furthermore, an increasing number of studies have demonstrated an association between OSA and cognitive impairment and CNS disease (19-22,24-26).

A previous study reported that OSA induces cognitive impairment via neuronal death in the hippocampus (45). Lim and Pack (46) reported that OSA alters BBB permeability by changing the expression levels of influx and efflux transporters, causing an acute leak via the tight junctions of the BBB. Halder and Milner (47) demonstrated that chronic hypoxia triggers BBB disruption and subsequent neurological dysfunction. In the present study, brain water content increased and BBB permeability was enhanced following $\mathrm{ICH}$ and further aggravated by subsequent OSA. Zolotoff et al (48) found that IH and sustained hypoxia can induce BBB 
A
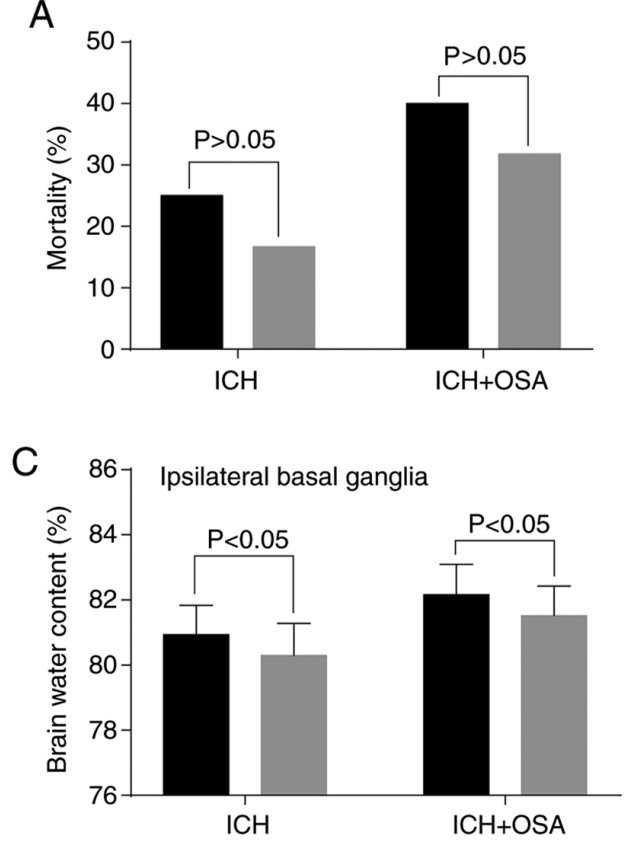

E

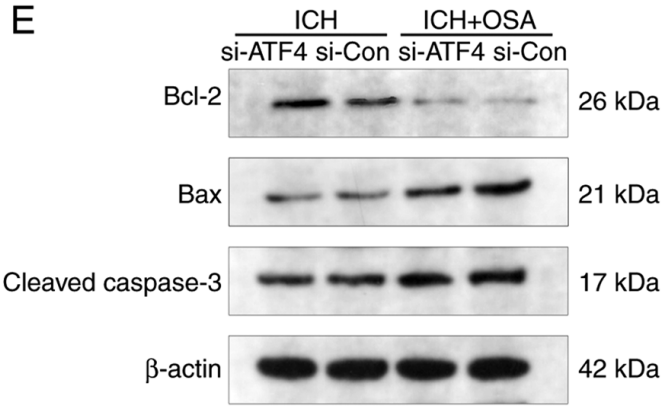

G

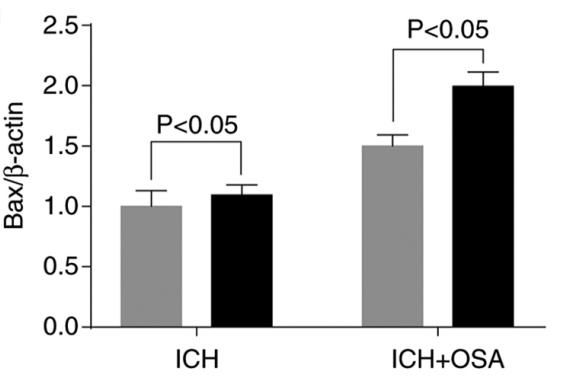

B

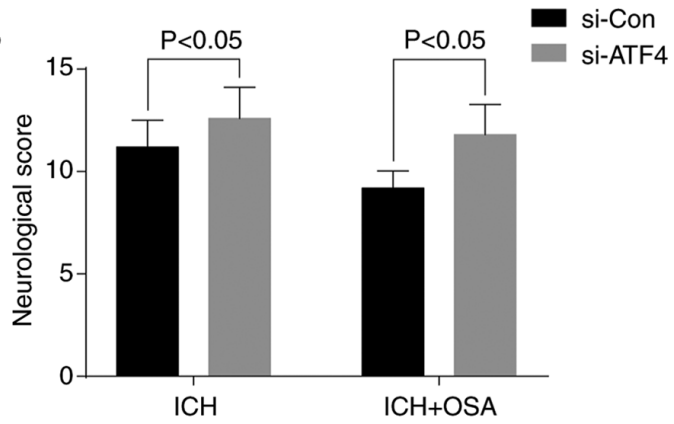

$\mathrm{D}$

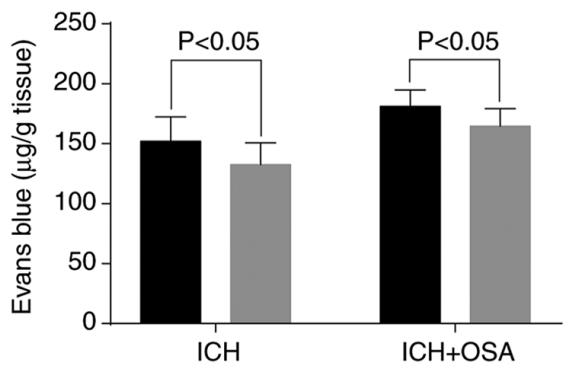

F

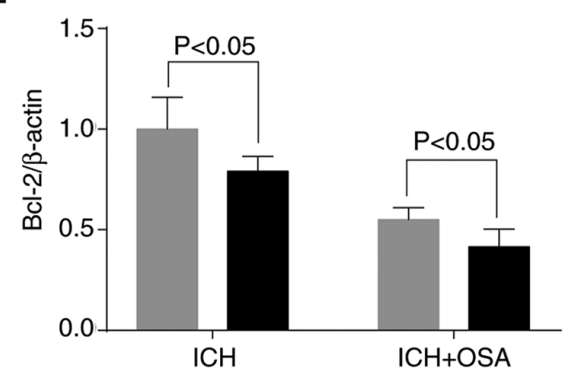

$\mathrm{H}$

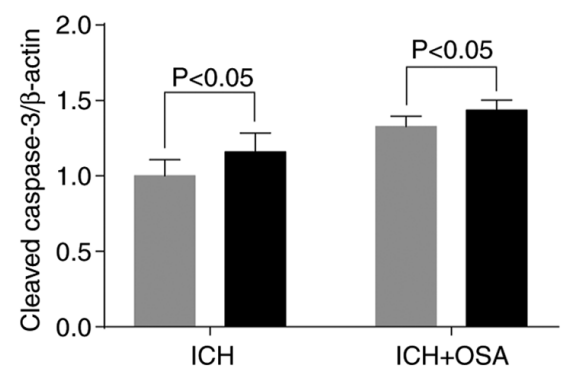

Figure 6. Knockdown of ATF4 reverses OSA-aggravated early brain injury following ICH. (A) Mortality rate was not significantly different compared with the ICH group. ATF4 knockdown reversed OSA-aggravated (B) neurological dysfunction (indicated by higher neurological score), (C) brain water content and (D) blood-brain barrier permeability. (E) Expression levels of apoptosis-associated proteins (Caspase-3, Bcl-2 and Bax) were detected by western blotting. Knockdown of ATF4 increased expression levels of (F) Bcl-2 and decreased those of $(\mathrm{G})$ Bax and $(\mathrm{H}) \mathrm{Caspase}-3$. Data are presented as the mean \pm SEM ( $\mathrm{n}=5$ ). ATF4, activating transcription factor 4; OSA, obstructive sleep apnea; ICH, intracerebral hemorrhage; si, small interfering; Con, control.

disruption and increase BBB permeability via production of ROS, Nrf2 and HIF-1 $\alpha$. ICH increases brain water content via BBB disruption, cerebral vasospasm and vascular endothelial dysfunction $(13,35)$. Hence, it was hypothesized that OSA combined with ICH increases brain water content and brain damage and that BBB permeability and vascular endothelial dysfunction serve an important role. The underlying molecular mechanisms are unclear and need further study.

Neuroinflammation is the primary pathophysiological change following cerebral hemorrhage; excessive neuroinflammatory response causes hippocampal neuronal apoptosis and EBI (27).
Molecular markers of neuroinflammation include pro-inflammatory cytokines IL- $1 \beta$, IL- 6 , TNF- $\alpha$ and NF- $\kappa$ B. Numerous studies have demonstrated that OSA induces cognitive deficits or brain damage, partly via oxidative stress and neuroinflammation, and that brain damage and neuronal apoptosis are alleviated if neuroinflammation is prevented (49-51). Gong et al (49) reported that OSA aggravates neuronal and microglial damage; damaged microglia release large amounts of proinflammatory cytokines, and an excessive inflammatory response leads to neurotoxicity and neuronal apoptosis by activating BNIP3-dependent mitophagy via the JNK/ERK signaling pathway. Additionally, 
OSA-induced nerve damage and stimulation of the interaction between toll-like receptor 2 and its aptamer MyD88 are associated with microglial aggregation, $\mathrm{NF}-\kappa \mathrm{B}$ activation, oxidative stress and elevated levels of inflammatory cytokines in mice (50). In the present study, expression levels of pro-inflammatory cytokines, including IL-1 $\beta$, IL-6, TNF- $\alpha$ and NF- $\kappa$ B, were increased significantly following ICH combined with OSA, thus aggravating EBI. The molecular mechanism may be associated with the ATF4/CHOP signaling pathway.

ATF4 serves different roles in different tissues, organs, and cells. In mammals, ATF4 is involved in a variety of physiological activities, such as eye development, bone formation, metabolism, energy balance, stress reaction, learning, memory and CNS disease $(52,53)$. Overexpressed ATF4 enters the nucleus and activates the transcription factor CHOP, which is involved in endoplasmic reticulum (ER) stress-induced neuronal apoptosis (54). ATF4 mRNA is expressed in all tissues, but ATF4 protein is only expressed at low levels under normal physiological conditions; the translation of ATF4 is upregulated by phosphorylation of the $\alpha$ subunit of eukaryotic initiation factor 2 (eIF2), which is triggered by ER and oxidative stress and amino acid deficiency (55). Baleriola et al (56) reported that the expression of ATF4 protein is increased in neuronal axons, as well as near amyloid plaques and in bead axons in the Alzheimer's disease brain. Inhibition of local translation and retrograde transport or knockdown of axonal ATF4 mRNA abolishes amyloid $\beta$-induced ATF4 transcriptional activity and cell loss (57). In a $\mathrm{CIH}$ rat model, a $\mathrm{H}_{2} / \mathrm{O}_{2}$ mixture inhibits ER stress-induced apoptosis via the PERK/eIF2 $\alpha /$ ATF4 signaling pathway and notably improves cardiac dysfunction and myocardial fibrosis (58). Furthermore, overexpression of ATF4 upregulates transcription of factors such as CHOP; this upregulates expression the apoptotic regulator mitochondrial apoptosis protein Bcl-2, which interacts with apoptotic regulators, activates the pro-apoptotic protein Bax and suppresses expression levels of anti-apoptotic gene Bcl-2 family proteins, thus promoting oxidative stress-induced nerve cell death (59). In the present study, expression levels of ATF4 and CHOP were increased significantly following $\mathrm{ICH}$ and further aggravated following OSA; this was associated with increased neuronal death and EBI. The present data showed that neuronal death and EBI following ICH/OSA were partially prevented following ATF4 knockdown. To the best of our knowledge, however, there have been no reports concerning the regulation of the downstream molecular pathway of CHOP following ICH/OSA and the exact mechanism requires further investigation.

In conclusion, OSA aggravated neurological dysfunction, brain edema, neuroinflammation and neuronal injury following ICH by promoting ER stress-associated apoptosis and neuroinflammation in hippocampal neurons. The mechanisms underlying these effects involved activation of ATF4/CHOP-mediated ER stress and apoptosis. Additionally, further investigations into the effects of OSA treatment combined with ICH in the clinic are required.

\section{Acknowledgements}

Not applicable.

\section{Funding}

No funding was received.

\section{Availability of data and materials}

The datasets used and/or analyzed during the current study are available from the corresponding author on reasonable request.

\section{Authors' contributions}

WF wrote the manuscript. WF, WJ, XF and YW performed the experiments and prepared the figures. WF and YW confirm the authenticity of all the raw data. XC and YW designed the study and revised the manuscript. All authors read and approved the final manuscript.

\section{Ethics approval and consent to participate}

The present study was approved by the Ethics Committee of the Wuxi Clinical College of Anhui Medical University (approval no. YXLL-2020-112; Wuxi, China).

\section{Patient consent for publication}

Not applicable.

\section{Competing interests}

The authors declare that they have no competing interests.

\section{References}

1. van Asch CJ, Luitse MJ, Rinkel GJ, van der Tweel I, Algra A and Klijn CJ: Incidence, case fatality, and functional outcome of intracerebral haemorrhage over time, according to age, sex, and ethnic origin: A systematic review and meta-analysis. Lancet Neurol 9: 167-176, 2010

2. Zhang Y, Zhang X, Wei Q, Leng S, Li C, Han B, Bai Y, Zhang H and Yao H: Activation of sigma-1 receptor enhanced pericyte survival via the interplay between apoptosis and autophagy: Implications for blood-brain barrier integrity in stroke. Transl Stroke Res 11: 267-287, 2020

3. Zhang Z, Cho S, Rehni AK, Quero HN, Dave KR and Zhao W: Automated assessment of hematoma volume of rodents subjected to experimental intracerebral hemorrhagic stroke by bayes segmentation approach. Transl Stroke Res 11: 789-798, 2020.

4. Gross BA, Jankowitz BT and Friedlander RM: Cerebral intraparenchymal hemorrhage: A review. JAMA 321: 1295-1303, 2019.

5. Wu X, Luo J, Liu H, Cui W, Guo K, Zhao L, Bai H, Guo W, Guo H, Feng D and Qu Y: Recombinant adiponectin peptide ameliorates brain injury following intracerebral hemorrhage by suppressing astrocyte-derived inflammation via the inhibition of Drp1-mediated mitochondrial fission. Transl Stroke Res 11: 924-939, 2020.

6. Hanley DF, Thompson RE, Rosenblum M, Yenokyan G, Lane K, McBee N, Mayo SW, Bistran-Hall AJ, Gandhi D, Mould WA, et al: Efficacy and safety of minimally invasive surgery with thrombolysis in intracerebral haemorrhage evacuation (MISTIE III): A randomised, controlled, open-label, blinded endpoint phase 3 trial. Lancet 393: 1021-1032, 2019.

7. Chen J, Zhu J, He J, Wang Y, Chen L, Zhang C, Zhou J and Yang L: Ultra-early microsurgical treatment within 24 h of SAH improves prognosis of poor-grade aneurysm combined with intracerebral hematoma. Oncol Lett 11: 3173-3178, 2016.

8. Chen J, Wang Y, Wu J, Yang J, Li M and Chen Q: The potential value of targeting ferroptosis in early brain injury after acute CNS disease. Front Mol Neurosci 13: 110, 2020. 
9. Adeoye $\mathrm{O}$ and Broderick JP: Advances in the management of intracerebral hemorrhage. Nat Rev Neurol 6: 593-601, 2010.

10. Mendelow AD, Gregson BA, Rowan EN, Murray GD, Gholkar A and Mitchell PM; STICH II Investigators: Early surgery versus initial conservative treatment in patients with spontaneous supratentorial lobar intracerebral haematomas (STICH II): A randomised trial. Lancet 382: 397-408, 2013.

11. Zhou Y, Wang Y, Wang J, Anne Stetler R and Yang QW: Inflammation in intracerebral hemorrhage: From mechanisms to clinical translation. Prog Neurobiol 115: 25-44, 2014.

12. Xue $\mathrm{M}$ and Yong VW: Neuroinflammation in intracerebral haemorrhage: Immunotherapies with potential for translation. Lancet Neurol 19: 1023-1032, 2020.

13. Chen JH, Yang LK, Chen L, Wang YH, Wu Y, Jiang BJ, Zhu J and Li PP: Atorvastatin ameliorates early brain injury after subarachnoid hemorrhage via inhibition of AQP4 expression in rabbits. Int J Mol Med 37: 1059-1066, 2016.

14. Bao WD, Zhou XT, Zhou LT, Wang F, Yin X, Lu Y, Zhu LQ and Liu D: Targeting miR-124/Ferroportin signaling ameliorated neuronal cell death through inhibiting apoptosis and ferroptosis in aged intracerebral hemorrhage murine model. Aging Cell 19: e13235, 2020.

15. Gautam J, Xu L, Nirwane A, Nguyen B and Yao Y: Loss of mural cell-derived laminin aggravates hemorrhagic brain injury. J Neuroinflammation 17: 103, 2020 .

16. Karuppagounder SS, Alim I, Khim SJ, Bourassa MW, Sleiman SF, John R, Thinnes CC, Yeh TL, Demetriades M, Neitemeier S, et al: Therapeutic targeting of oxygen-sensing prolyl hydroxylases abrogates ATF4-dependent neuronal death and improves outcomes after brain hemorrhage in several rodent models. Sci Transl Med 8: 328ra329, 2016.

17. Chen J, Xuan Y, Chen Y, Wu T, Chen L, Guan H, Yang S, He J, Shi D and Wang Y: Netrin-1 alleviates subarachnoid haemorrhage-induced brain injury via the PPAR $\gamma / \mathrm{NF}-\mathrm{KB}$ signalling pathway. J Cell Mol Med 23: 2256-2262, 2019.

18. Zhou Y, Tao T, Liu G, Gao X, Gao Y, Zhuang Z, Lu Y, Wang H, $\mathrm{Li} \mathrm{W}, \mathrm{Wu} \mathrm{L}$, et al: TRAF3 mediates neuronal apoptosis in early brain injury following subarachnoid hemorrhage via targeting TAK1-dependent MAPKs and NF- $\kappa$ B pathways. Cell Death Dis 12: $10,2021$.

19. Chernyshev OY, Bir SC, Maiti TK, Patra DP, Sun H, Guthikonda B, Kelley RE, Cuellar H, Minagar A and Nanda A: The relationship between obstructive sleep apnea and ruptured intracranial aneurysms. J Clin Sleep Med 15: 1839-1848, 2019.

20. Mason RH, Ruegg G, Perkins J, Hardinge M, Amann-Vesti B, Senn O, Stradling JR and Kohler M: Obstructive sleep apnea in patients with abdominal aortic aneurysms: Highly prevalent and associated with aneurysm expansion. Am J Respir Crit Care Med 183: 668-674, 2011.

21. Zaremba S, Albus L, Schuss P, Vatter H, Klockgether T and Güresir E: Increased risk for subarachnoid hemorrhage in patients with sleep apnea. J Neurol 266: 1351-1357, 2019.

22. Geer JH, Falcone GJ, Vanent KN, Leasure AC, Woo D, Molano JR, Sansing LH, Langefeld CD, Pisani MA, Yaggi HK and Sheth KN: Obstructive sleep apnea as a risk factor for intracerebral hemorrhage. Stroke 52: 1835-1838, 2021.

23. Bir SC, Nanda A, Cuellar H, Sun H, Guthikonda B, Liendo C, Minagar A and Chernyshev OY: Coexistence of obstructive sleep apnea worsens the overall outcome of intracranial aneurysm: A pioneer study. J Neurosurg 128: 735-746, 2018

24. Pontes-Neto OM, Fernandes RM, Sander HH, da Silva LA Mariano DC, Nobre F, Simão G, de Araujo DB, dos Santos AC and Leite JP: Obstructive sleep apnea is frequent in patients with hypertensive intracerebral hemorrhage and is related to perihematoma edema. Cerebrovasc Dis 29: 36-42, 2010.

25. Orrù G, Storari M, Scano A, Piras V, Taibi R and Viscuso D: Obstructive sleep apnea, oxidative stress, inflammation and endothelial dysfunction-an overview of predictive laboratory biomarkers. Eur Rev Med Pharmacol Sci 24: 6939-6948, 2020

26. Hung MW, Tipoe GL, Poon AM, Reiter RJ and Fung ML: Protective effect of melatonin against hippocampal injury of rats with intermittent hypoxia. J Pineal Res 44: 214-221, 2008.

27. Chen J,Zhang C, Yan T, Yang L, Wang Y, Shi Z, Li M and Chen Q: Atorvastatin ameliorates early brain injury after subarachnoid hemorrhage via inhibition of pyroptosis and neuroinflammation. J Cell Physiol: Mar 31, 2021 (Epub ahead of print).

28. Wu X, Luo J, Liu H, Cui W, Guo W, Zhao L, Guo H, Bai H, Guo K, Feng D and Qu Y: Recombinant adiponectin peptide promotes neuronal survival after intracerebral haemorrhage by suppressing mitochondrial and ATF4-CHOP apoptosis pathways in diabetic mice via Smad3 signalling inhibition. Cell Prolif 53: e12759, 2020.
29. Lu Z, Wang Z, Yu L, Ding Y, Xu Y, Xu N, Li R, Tang J, Chen G and Zhang JH: GCN2 reduces inflammation by p-eIF2 $\alpha / A T F 4$ pathway after intracerebral hemorrhage in mice. Exp Neurol 313: $16-25,2019$.

30. National Research Council Institute for Laboratory Animal Research: In: Guide for the Care and Use of Laboratory Animals National Academies Press. National Academy of Sciences, Washington, DC, 1996.

31. Deng S, Sherchan P, Jin P, Huang L, Travis Z, Zhang JH, Gong Y and Tang J: Recombinant CCL17 enhances hematoma resolution and activation of CCR4/ERK/Nrf2/CD163 signaling pathway after intracerebral hemorrhage in mice. Neurotherapeutics 17 : 1940-1953, 2020

32. Li L, Ren F, Qi C, Xu L, Fang Y, Liang M, Feng J, Chen B, Ning $\mathrm{W}$ and Cao J: Intermittent hypoxia promotes melanoma lung metastasis via oxidative stress and inflammation responses in a mouse model of obstructive sleep apnea. Respir Res 19: 28 , 2018.

33. Chen JH, Wu T, Xia WY, Shi ZH, Zhang CL, Chen L, Chen QX and Wang YH: An early neuroprotective effect of atorvastatin against subarachnoid hemorrhage. Neural Regen Res 15: 1947-1954, 2020

34. Li G, Dong Y, Liu D, Zou Z, Hao G, Gao X, Pan P and Liang G NEK7 coordinates rapid neuroinflammation after subarachnoid hemorrhage in mice. Front Neurol 11: 551, 2020.

35. Chen JH, Wu T, Yang LK, Chen L, Zhu J, Li PP, Hu X and Wang YH: Protective effects of atorvastatin on cerebral vessel autoregulation in an experimental rabbit model of subarachnoid hemorrhage. Mol Med Rep 17: 1651-1659, 2018.

36. Livak KJ and Schmittgen TD: Analysis of relative gene expression data using real-time quantitative PCR and the 2(-Delta Delta $\mathrm{C}(\mathrm{T})$ ) method. Methods 25: 402-408, 2001.

37. Nguyen JQN, Resnick CM, Chang YH, Hansen RM, Fulton AB, Moskowitz A, Calabrese CE and Dagi LR: Impact of obstructive sleep apnea on optic nerve function in patients with craniosynostosis and recurrent intracranial hypertension. Am J Ophthalmol 207: 356-362, 2019.

38. Wei C, Guo S, Liu W, Jin F, Wei B, Fan H, Su H, Liu J, Zhang N, Fang D, et al: Resolvin D1 ameliorates inflammation-mediated blood-brain barrier disruption after subarachnoid hemorrhage in rats by modulating A20 and NLRP3 inflammasome. Front Pharmacol 11: 610734, 2020.

39. Deng S, Liu S, Jin P, Feng S, Tian M, Wei P, Zhu H, Tan J, Zhao F and Gong Y: Albumin reduces oxidative stress and neuronal apoptosis via the ERK/Nrf2/HO-1 pathway after intracerebral hemorrhage in rats. Oxid Med Cell Longev 2021: 8891373, 2021.

40. Prabhakar NR, Peng YJ and Nanduri J: Hypoxia-inducible factors and obstructive sleep apnea. J Clin Invest 130: 5042-5051, 2020.

41. Tan J, Xing H, Sha S, Li J, Miao Y and Zhang Q: Analysis of circulating microvesicles levels and effects of associated factors in elderly patients with obstructive sleep apnea. Front Aging Neurosci 13: 609282, 2021.

42. Strausz S, Ruotsalainen S, Ollila HM, Karjalainen J, Kiiskinen T, Reeve M, Kurki M, Mars N, Havulinna AS, Luonsi E, et al: Genetic analysis of obstructive sleep apnoea discovers a strong association with cardiometabolic health. Eur Respir J 57: 2003091, 2021

43. Li J, McEvoy RD, Zheng D, Loffler KA, Wang X, Redline S, Woodman RJ and Anderson CS: Self-reported snoring patterns predict stroke events in high-risk patients with osa: Post hoc analyses of the save study. Chest 158: 2146-2154, 2020.

44. Yu LM, Zhang WH, Han XX, Li YY, Lu Y, Pan J, Mao JQ, Zhu LY, Deng JJ, Huang W and Liu YH: Hypoxia-induced ROS contribute to myoblast pyroptosis during obstructive sleep apnea via the NF- $\kappa \mathrm{B} / \mathrm{HIF}-1 \alpha$ signaling pathway. Oxid Med Cell Longev 2019: 4596368, 2019.

45. Zhu Y, Fenik P, Zhan G, Mazza E, Kelz M, Aston-Jones G and Veasey SC: Selective loss of catecholaminergic wake active neurons in a murine sleep apnea model. J Neurosci 27: 10060-10071, 2007.

46. Lim DC and Pack AI: Obstructive sleep apnea and cognitive impairment: Addressing the blood-brain barrier. Sleep Med Rev 18: 35-48, 2014

47. Halder SK and Milner R: Mild hypoxia triggers transient blood-brain barrier disruption: A fundamental protective role for microglia. Acta Neuropathol Commun 8: 175, 2020.

48. Zolotoff C, Voirin AC, Puech C, Roche F and Perek N: Intermittent hypoxia and its impact on Nrf2/HIF-1 $\alpha$ expression and $\mathrm{ABC}$ transporters: $\mathrm{An}$ in vitro human blood-brain barrier model study. Cell Physiol Biochem 54: 1231-1248, 2020. 
49. Gong LJ, Wang XY, Gu WY and Wu X: Pinocembrin ameliorates intermittent hypoxia-induced neuroinflammation through BNIP3-dependent mitophagy in a murine model of sleep apnea. J Neuroinflammation 17: 337, 2020.

50. Deng Y, Liu K, Pan Y, Ren J, Shang J, Chen L and Liu H: TLR2 antagonism attenuates the hippocampal neuronal damage in a murine model of sleep apnea via inhibiting neuroinflammation and oxidative stress. Sleep Breath 24: 1613-1621, 2020.

51. Johnson SM, Randhawa KS, Epstein JJ, Gustafson E, Hocker AD, Huxtable AG, Baker TL and Watters JJ: Gestational intermittent hypoxia increases susceptibility to neuroinflammation and alters respiratory motor control in neonatal rats. Respir Physiol Neurobiol 256: 128-142, 2018.

52. Wang C and Guo F: Effects of activating transcription factor 4 deficiency on carbohydrate and lipid metabolism in mammals. IUBMB Life 64: 226-230, 2012.

53. Aimé P, Karuppagounder SS, Rao A, Chen Y, Burke RE, Ratan RR and Greene LA: The drug adaptaquin blocks ATF4/CHOP-dependent pro-death Trib3 induction and protects in cellular and mouse models of Parkinson's disease. Neurobiol Dis 136: 104725, 2020.

54. Galehdar Z, Swan P, Fuerth B, Callaghan SM, Park DS and Cregan SP: Neuronal apoptosis induced by endoplasmic reticulum stress is regulated by ATF4-CHOP-mediated induction of the Bcl-2 homology 3-only member PUMA. J Neurosci 30: $16938-16948,2010$.
55. Harding HP, Zhang Y, Zeng H, Novoa I, Lu PD, Calfon M, Sadri N, Yun C, Popko B, Paules R, et al: An integrated stress response regulates amino acid metabolism and resistance to oxidative stress. Mol Cell 11: 619-633, 2003.

56. Baleriola J, Walker CA, Jean YY, Crary JF, Troy CM, Nagy PL and Hengst U: Axonally synthesized ATF4 transmits a neurodegenerative signal across brain regions. Cell 158: 1159-1172, 2014.

57. Liu Y: Hydrogen peroxide induces nucleus pulposus cell apoptosis by ATF4/CHOP signaling pathway. Exp Ther Med 20: 3244-3252, 2020

58. Zhao YS, An JR, Yang S, Guan P, Yu FY, Li W, Li JR, Guo Y, Sun ZM and Ji ES: Hydrogen and oxygen mixture to improve cardiac dysfunction and myocardial pathological changes induced by intermittent hypoxia in rats. Oxid Med Cell Longev 2019: $7415212,2019$.

59. Lange PS, Chavez JC, Pinto JT, Coppola G, Sun CW, Townes TM, Geschwind DH and Ratan RR: ATF4 is an oxidative stress-inducible, prodeath transcription factor in neurons in vitro and in vivo. J Exp Med 205: 1227-1242, 2008.

(7)(9) This work is licensed under a Creative Commons

cc) Attribution-NonCommercial-NoDerivatives 4.0 International (CC BY-NC-ND 4.0) License. 